\title{
Operative Hysteroscopy in Infertility Patients and Its Complications
}

\author{
Kriti Sahu' ${ }^{1}$, Beena Kumari² \\ 1Department of Obstetrics and Gynaecology, Dr. Babasaheb Ambedkar Memorial Hospital, \\ Central Railway, Mumbai, Maharashtra, India. ${ }^{2}$ Department of Obstetrics and Gynaecology, Dr. \\ Babasaheb Ambedkar Memorial Hospital, Central Railway, Mumbai, Maharashtra, India.
}

\section{ABSTRACT}

\section{BACKGROUND}

The problem of infertility is increasing over the years and so are the couples seeking medical help for it. Estimates of infertility in India goes to around 10 - 15 percent. Hence evaluation of the couple with the help of history and accompanying investigations are foremost required. Various modalities are available for identifying the cause. One such cause is abnormalities in the uterine cavity. This step is important because its inner layer, called endometrium is responsible for implantation of the embryo in the blastocyst stage. For such assessment of the uterine cavity, the gold standard procedure is the diagnostic hysteroscopy. It involves direct visualization of the cavity and simultaneous correction of the intrauterine pathology. Hysteroscopy is a simple procedure with low risk of complication; moreover, complications occur with operative procedures most commonly. This study aims to evaluate these complications and critically appraise the changes in trend of complications.

\section{METHODS}

This is an observational study. Study included 32 patients with infertility undergoing operative hysteroscopy at obstetrics and gynaecology department in our hospital.

\section{RESULTS}

Among the 32 patients, the most common hysteroscopic procedure was lateral wall metroplasty (40.6\%) followed by septal resection (25\%). Polypectomy, adhesiolysis and tubal cannulation. Complication rate was 6.25 percent, among which post-op infection and bleeding per vaginum were the most important.

\section{CONCLUSIONS}

Intraoperative complications found in our study are comparable to other studies and largely preventable. Hence operative hysteroscopy is a safe procedure.

\section{KEY WORDS}

Operative Hysteroscopy, Lateral Wall Metroplasty, Septal Resection
Corresponding Author: Dr. Kriti Sahu,

Room No. 3, $4^{\text {th }}$ Floor, Dr. Babasaheb Ambedkar Memorial Hospital, Central Railway, Near Ranibaug, Byculla East, Mumbai, Maharashtra, India.

E-mail: kriti.sahu2001@gmail.com

DOI: $10.14260 / \mathrm{jemds} / 2020 / 411$

Financial or Other Competing Interests: None.

How to Cite This Article:

Sahu K, Kumari B.

Operative hysteroscopy in infertility patients and its complications. J. Evolution Med. Dent. Sci. 2020;9(26):1890-1894, DOI: $10.14260 / \mathrm{jemds} / 2020 / 411$

Submission 02-04-2019,

Peer Review 26-05-2020,

Acceptance 05-06-2020,

Published 29-06-2020.

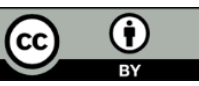




\section{BACKGROUND}

According to the definition of infertility, it is the inability of a couple to conceive after 1 year of unprotected intercourse of reasonable frequency. It is of two types: as primary infertility when there is no prior conception and secondary infertility, when there is infertility following at least one prior conception, irrespective of the outcome. Infertility is increasing day by day owning to late marriages and couples seeking medical help for it are also increasing dramatically. ${ }^{1}$ Overall its incidence varies between 5-15 percent. $^{2}$ In India estimates of infertility are around 10-15 percent. $^{3}$

Usually evaluation for infertility for any couple starts when they fail to conceive in 1 year, but in several scenarios like age more than 35 years, may prompt earlier intervention. ${ }^{4}$ Infertility consultation includes careful history taking, examinations and various investigations for identifying the cause. One such cause is abnormalities in the uterine cavity. This step is important because its inner layer, called endometrium is responsible for implantation of the embryo in the blastocyst stage. Implantation phase is established by the complex interaction between the blastocyst stage of embryo and the endometrium. ${ }^{5}$ Hence assessing the cavity to rule out possible abnormalities is required. For such assessment of the uterine cavity, the gold standard procedure is the diagnostic hysteroscopy. Among the women seeking treatment for infertility, major uterine cavity abnormalities can be found in 10 to 15 percent of the cases; they usually have endometrial polyps. ${ }^{6}$

Preferably transvaginal ultrasonography (TVS), is used for the screening of possible endometrial or uterine cavity abnormalities in the work-up of infertile women. Further extended evaluation can be done with other procedures such as, HSG (hysterosalpingography), SIS (saline infusion sonography) and diagnostic hysteroscopy. Hysteroscopy is called diagnostic if the procedure id done just for evaluation of the uterine cavity. If any pathology is observed during the procedure or is suspected on TVS and requires further treatment procedure, then hysteroscopic procedure is called an operative hysteroscopy. Often in practice if diagnostic hysteroscopy confirms the presence of pathology then it will be followed by an operative hysteroscopy. ${ }^{7}$

Hysteroscopy gives a direct visualisation of the uterine cavity using a rigid, semi-rigid or flexible endoscope. For this reason, it's the gold standard procedure for assessment of the uterine cavity. Also, as an added advantage with diagnostic hysteroscopy, direct visualization and the treatment of intrauterine pathology can be done in the same setting. ${ }^{8}$ This also improves cost-effectiveness.

The most common procedures performed in operative hysteroscopy for infertility are septoplasty, that is, resection of uterine septa, lysis of intrauterine synechiae, hysteroscopic metroplasty and resection of the submucous leiomyomata. ${ }^{9}$ several hypotheses formulated and studies have been done in the literature of how various endometrial pathologies like endometrial polyps ${ }^{10,11,12,13}$ submucous fibroids, ${ }^{14,15}$ intrauterine adhesions ${ }^{16}$ and uterine septum ${ }^{17}$ likely hamper embryo implantation; however, still the exact mechanisms by which such major uterine cavity abnormalities affect this essential reproductive process are not clearly understood.

According to literature, most common acquired uterine cavity abnormality is endometrial polyp, which is benign endometrial stalk-like mass with vascularity, protruding into the uterine cavity. Further, some observational studies suggest higher pregnancy rates after the removal of polyps, submucous fibroids, uterine septum or intrauterine adhesions by hysteroscopy.

Operative hysteroscopy procedure requires adequate visualization of uterine cavity through a media, which is a continuous fluid circulation using an in and outflow channel. Diagnostic hysteroscopy can be done using a smaller scope in office setting and is termed office hysteroscopy using local anaesthesia and fluid distension media, while on the other hand more complex procedures like operative hysteroscopies are performed as day-care surgery under general anaesthesia.18 they also require complex instruments, specialized trained surgeon and appropriate knowledge of management of complications. ${ }^{19}$ there are few complications in hysteroscopy. These can occur when proper techniques of the procedure are not followed, improper using of instrument, lack of technical expertise and ignoring the contraindications. Also, proper selection of patients is important.

Causes of common complications include

Intra-op

1. Anaesthesia related.

2. Improper positioning of the patient.

3. Problems due to distension media like fluid overload.

4. Surgical complications:

- Uterine perforation.

- Haemorrhage.

Post-op

1. Post-operative infection.

2. Uterine adhesion formation.

3. Non- resolution of the presenting symptoms. ${ }^{20}$

Operative hysteroscopy can lead to complications more commonly and are potentially more serious. Also, early recognition would help the prevention of the complications as imminent steps can be taken and progression to adverse events can be prevented. Many of these serious complications can be avoided by taking proper precautions. ${ }^{21}$ There is recent decline in complications during hysteroscopy due to better technology and improvement in hysteroscopy techniques. The learning curve is improving as more and more centres of training in minimal invasive surgeries are being started. There has been a well-established role of office hysteroscopy in infertility. ${ }^{22}$ In terms of cost-effectiveness, patients of infertility are recommended to undergo uterine cavity evaluation by a hysteroscopy and treatment of the probable causes in the same setting. Hence knowing the complication rate becomes of utmost importance.

The aim of this study was to evaluate the complications of operative hysteroscopy.

\section{METHODS}

This was an observational study and conducted at the Department Obstetrics and Gynaecology, Dr. Babasaheb 
Ambedkar Memorial Hospital Central Railway, Mumbai. Sampling was consecutive and participants were observed and interviewed for the purpose of study between May 2018 and October 2018 (duration 6 months).

\section{Ethics}

The study protocol was presented to institutional ethics committee and was approved before commencement of study. Patients were briefed about the study, assured confidentiality and written informed consent was obtained from willing participants.

\section{Study Sample}

Reproductive age group women 18 -45 years that underwent operative hysteroscopic procedures from May 2018 to October 2018 were included in study. Hence study included 32 patients with infertility undergoing operative hysteroscopy at Obstetrics and Gynaecology department in our hospital during the 6 months period. Various operative hysteroscopy procedures in patient of infertility were noted. Complications occurring in these operative hysteroscopy procedures were compared with the rate of complications reported in other studies.

\section{Data Collection}

Data was collected from

1. Case record forms to record clinical details.

2. Observations- intra-operatively and post-operatively of the patients

\section{Statistical Analysis}

The collected data obtained was analysed by using software SPSS 20.0. Qualitative data analysis was done using frequency and percentage. Associations were documented on percentage basis as follows

1. Abnormal hysteroscopic findings and operative procedures done.

2. Complications in operative hysteroscopy.

3. Comparison and analysis of occurrence of complications in operative hysteroscopies in other studies.

\section{RESULTS}

\begin{tabular}{|cc|}
\hline Hysteroscopic Procedure Done & No. of Patients \% (n=32) \\
Polypectomy & $15.6(5)$ \\
Adhesiolysis & $6.25(2)$ \\
Metroplasty & $40.6(13)$ \\
Septoplasty & $25(8)$ \\
Tubal cannulation & $9.3(3)$ \\
Myomectomy & $3.1(1)$ \\
\hline Table 1. Common Hysteroscopic Procedures and Their Percentages
\end{tabular}

\begin{tabular}{|cc|}
\hline Complications Reported & Percentage $\mathbf{n}=\mathbf{3 2}$ \\
Total complication rate & $6.2 \%(2)$ \\
Fluid overload & 0 \\
Uterine perforation & 0 \\
Bladder injury & 0 \\
Bowel injury & 0 \\
Post op infection & $3.1 \%(1)$ \\
Post op bleeding per vaginum & $3.1 \%(1)$ \\
\hline Table 2. Complications in Operative Hysteroscopy \\
\hline
\end{tabular}

\begin{tabular}{|c|c|c|c|c|c|c|}
\hline $\begin{array}{l}\text { Complications } \\
\text { Reported }\end{array}$ & $\begin{array}{c}\text { Scottish } \\
\text { Audit } \\
\text { (1995) } \\
\text { n=978 }\end{array}$ & $\begin{array}{c}\text { PROPST } \\
\text { et al } \\
(2000) \\
n=925\end{array}$ & $\begin{array}{c}\begin{array}{c}\text { Jansen } \\
\text { et al } \\
(2000)\end{array} \\
n=13600\end{array}$ & $\begin{array}{c}\text { Pasini } \\
\text { et al } \\
(2001) \\
n=697\end{array}$ & $\begin{array}{c}\text { Sandhya } \\
\text { Deora } \\
\text { et al (2018) } \\
\mathbf{n}=327\end{array}$ & $\begin{array}{c}\text { Our } \\
\text { Study } \\
\mathrm{n}=32\end{array}$ \\
\hline $\begin{array}{l}\text { Total complication } \\
\text { rate }\end{array}$ & $12 \%$ & $2.7 \%$ & $0.28 \%$ & $13.6 \%$ & $0.9 \%$ & $6.2 \%$ \\
\hline Fluid overload & $01 \%$ & $0.76 \%$ & $0.2 \%$ & $05 \%$ & $0.3 \%$ & - \\
\hline Uterine perforation & $01 \%$ & $0.4 \%$ & $0.76 \%$ & $1.7 \%$ & $0.76 \%$ & - \\
\hline Bladder injury & - & - & - & - & - & - \\
\hline Bowel injury & - & - & - & - & - & - \\
\hline Post op infection & - & - & - & - & - & $3.1 \%$ \\
\hline $\begin{array}{l}\text { Post op bleeding per } \\
\text { vaginum }\end{array}$ & - & - & - & - & - & $3.1 \%$ \\
\hline
\end{tabular}

In our series of 32 patients most common hysteroscopic procedure was lateral wall metroplasty $(40.6 \%)$ followed by septal resection (25\%). Polypectomy, adhesiolysis and tubal cannulation were other common hysteroscopic procedures. (Table 1) Complication rate was reported as $6.25 \%$. Post-op infection and bleeding per-vaginum were the most important complications in our study. Total complication rate and rate of other complications were comparable to the rates reported in other series.

\section{DISCUSSION}

In recent surgical practice office hysteroscopy has a special place but its role is much limited to older age group of patients. In younger patients of infertility, it is still under debate. Much of it, may be due to use of energy sources inside the body or lack of knowledge and experience with such techniques. Even after many trials and literature showing its effectiveness the consensus in infertility is yet to be formed. Many surgeons still resort to non-invasive techniques like ultrasonography over hysteroscopy and further after recurrent failures of conceptions finally hysteroscopy is done, and abnormality is detected. The advancement in reproductive medicine over few years is tremendous and more shocking is the acceptance and wide availability of these techniques. Following this, reproductive specialist are turning towards hysteroscopies and its utility in infertility. Studies have been done illustrating its effectiveness and complications.

A Scottish Hysteroscopy Audit Group study, in 1995, were among the earlier studies on the utility of hysteroscopy. They conducted 978 hysteroscopic procedures of which 629 resections, 314 laser ablations, 35 roller-ball ablations. They reported total 120 complications (12\%) which included 61 cases of fluid overload; 11 perforations, of which 6 were due to use of resectoscope; 35 cases of excessive bleeding per vaginum. 3 patients underwent hysterectomies due to these complications and one patient died due to septicaemia. ${ }^{23}$

Propst et al conducted study in 925 women and reported a complication rate of $2.7 \%$. Among these specific complications included excessive fluid absorption, uterine perforation, postoperative infection and termination of procedure due to inadequate view or inability to dilate the cervix. Four patients were kept for overnight observation or antibiotic treatment. No Laparoscopy or laparotomy was done because of a complication, nor were there any instances of haemorrhage, bladder or bowel injury. The highest risk for complications were in myomectomies and septal resections. ${ }^{24}$

A Multicentric study was conducted in 82 hospitals by Jansen et al in 1997. A total of 13600 hysteroscopic 
procedures were performed, out of which complication rate reported was $0.28 \%$. Complications in diagnostic procedures were around $0.13 \%$ (comparatively lower than operative procedures). Commonest complications were Fluid overloads of distention medium (rate $0.20 \%$ ) and uterine perforation (rate $0.76 \%$ ) of which approximately half the perforations were entry-related. Various surgeries related complication rate were: intrauterine adhesiolysis $4.48 \%$, endometrium resection $0.81 \%$, myomectomy $0.75 \%$, and polypectomy $0.38 \% .25$

In another study conducted by the Pasini et al in 2001, intraoperative complications of 697 patients were studied. Total complication rate reported was $13.6 \%$ (95 patients). Among these were uterine perforations 1.7\%, intraoperative haemorrhages $6.9 \%$ and fluid overload 5\%. Out of 12 perforations 4 occurred during cervical dilation. Procedures were stopped due to inadequate distention of the uterine cavity. There were no vaginal or intraperitoneal haemorrhage observed in the study. The tip of the electrical source caused 8 out of 12 perforations. Following which the operative hysteroscopies were immediately stopped and the further consequences were reported, that is, 6 diagnostic laparoscopies, 1 laparotomy and 2 hysterectomies. ${ }^{21}$

In another study of Sandhya Deora et al (2018), complication rate was 3 out of 327 patients $(0.9 \%)$. Common among them were: $2(0.6 \%)$ uterine perforations, and 1 $(0.3 \%)$ excessive hypotonic fluid absorptions. Total complication rate and rate of other complications were comparable to the rates reported in other series. One uterine perforation occurred during septal lysis in a case of complete uterine septum. Second perforation occurred in a case of resection of submucous myoma while using a bipolar resectoscope. In both cases procedure was terminated and patient managed conservatively. One case of fluid overload occurred in a case of resection of submucous myoma. Postoperatively, patient complained of headache, dizziness and vomiting. Patient was managed conservatively with diuretics and correction of electrolyte imbalance. ${ }^{26}$ Abnormal findings diagnosed at Hysteroscopy includes intrauterine synechiae, Mullerian fusion defects, Endometrial polyp, Submucous myoma, Intra uterine septum. ${ }^{27,28}$

According to our study most common hysteroscopic procedure done was lateral wall metroplasty (40.6\%) followed by septoplasty (25\%) and polypectomy (15.6\%). Other procedures done were adhesiolysis, tubal cannulation and myomectomy. (Table 1)

Intraoperative complications found in our study were comparable to the percentages of complications in other studies and largely preventable. (TABLE 3) One patient had excessive bleeding per vaginum in post-operative period following resection of submucous fibroid, which was managed conservatively. Another patient had mild fever on post-operative day 1 , which subsided with antibiotics and antipyretics. The overall complication rate was 6.2 (Table 2) The recent trend of decrease in complications of operative hysteroscopy is due to use of cold scissors for procedures like lateral metroplasty and septoplasty. Scissors are preferred over the resectoscope because there is no thermal damage to the endometrium. Duffy et al. ${ }^{29}$ demonstrated a narrow zone of thermal necrosis in response to endometrial resection. In patients of infertility further use of resectoscope would destroy the endometrium and prevent implantation hence use of energy source like bipolar and unipolar resectoscope have been avoided in our study. But this exposes the patient to bleeding, nevertheless careful selection of cases and pre-op evaluation to be done carefully if vascular pathology like myoma or polyp is detected. Also, as the technical advances have occurred, overall expertise for operative hysteroscopy has increased which leads to decrease in rate of complications.

\section{CONCLUSIONS}

Hysteroscopy has an important role in the treatment of uterine factor for infertility. Operative hysteroscopy had a low rate of morbidity and satisfactory efficacy with rapid postoperative recovery. Hence operative hysteroscopy is a safe procedure. Further studies on the effect of hysteroscopy on uterus and fertility outcomes needs to be studied.

\section{REFERENCES}

[1] Aral SO, Cates W. The increasing concern with infertility. Why now? JAMA 1983;250(17):2327-31.

[2] Shaw W, Howkins J, Bourne GL. Shaw's textbook of gynaecology. $16^{\text {th }}$ edn. New Delhi: Elsevier India Private Limited 2015.

[3] Adamson GD, De Mouzon J, Ishihara 0, et al. International Committee for Monitoring Assisted Reproductive Technology (ICMART) and the World Health Organization (WHO) revised glossary of ART. Fertil Steril 2009;92(5):1520-4.

[4] Hoffman B, Schorge J, Bradshaw K. William's gynecology. $3^{\text {rd }}$ edn. New York: McGraw-Hill Publication 2016.

[5] Singh M, Chaudhry P, Asselin E. Bridging endometrial receptivity and implantation: network of hormones, cytokines, and growth factors. J Endocrinol 2011;210(1):5-14.

[6] Wallach EE. The uterine factor in infertility. Fertil Steril 1972;23(2):138-58.

[7] Bosteels J, van Wessel S, Weyers S, et al. Hysteroscopy for treating subfertility associated with suspected major uterine cavity abnormalities. Cochrane Database Syst Rev 2018(12):CD009461.

[8] Yanaihara A, Yorimitsu T, Motoyama H, et al. Location of endometrial polyp and pregnancy rate in infertility patients. Fertil Steril 2008;90(1):180-2.

[9] Fernandez H, Gervaise A, de Tayrac R. Operative hysteroscopy for infertility using normal saline solution and a coaxial bipolar electrode: a pilot study. Hum Reprod 2000;15(8):1773-5.

[10] Silberstein T, Saphier O, van Voorhis BJ, et al. Endometrial polyps in reproductive-age fertile and infertile women. Isr Med Assoc J 2006;8(3):192-5.

[11] Yanaihara A, Yorimitsu T, Motoyama H, et al. Location of endometrial polyp and pregnancy rate in infertility patients. Fertil Steril 2008;90(1):180-2.

[12] Pérez-Medina T, Bajo-Arenas J, Salazar F, et al. Endometrial polyps and their implication in the pregnancy rates of patients undergoing intrauterine 
insemination: a prospective, randomized study. Hum Reprod 2005;20(6):1632-5.

[13] Kalampokas T, Tzanakaki D, Konidaris S, et al. Endometrial polyps and their relationship in the pregnancy rates of patients undergoing intrauterine insemination. Clin Exp Obstet Gynecol 2012;39(3):299302.

[14] Pritts EA, Parker WH, Olive DL. Fibroids and infertility: an updated systematic review of the evidence. Fertil Steril 2009;91(4):1215-23.

[15] Jayakrishnan K, Menon V, Nambiar D. Submucous fibroids and infertility: effect of hysteroscopic myomectomy and factors influencing outcome. J Hum Reprod Sci 2013;6(1):35-9.

[16] Yu D, Li TC, Xia E, et al. Factors affecting reproductive outcome of hysteroscopic adhesiolysis for Asherman's syndrome. Fertil Steril 2008;89(3):715-22.

[17] Fedele L, Bianchi S, Marchini M, et al. Residual uterine septum of less than $1 \mathrm{~cm}$ after hysteroscopic metroplasty does not impair reproductive outcome. Hum Reprod 1996;11(4):727-9.

[18] Clark TJ, Daniels J, Gray R, et al. A randomised controlled trial of outpatient polyp treatment for abnormal uterine bleeding. Centre for Reviews and Dissemination 2010.

[19] Campo R, Molinas CR, Rombauts L, et al. Prospective multicentre randomized controlled trial to evaluate factors influencing the success rate of office diagnostic hysteroscopy. Human Reproduction 2005;20(1):258-63.
[20] Pritts EA, Parker WH, Olive DL. Fibroids and infertility: an updated systematic review of the evidence. Fertil Steril 2009;91(4):1215-23.

[21] Pasini A, Belloni C. Intraoperative complications of 697 consecutive operative hysteroscopies. Minerva Ginecol 2001;53(1):13-20.

[22] Barati M, Zargar M, Masihi S, et al. Office hysteroscopy in infertility. Int J Fertil Steril 2009;3(1):17-20.

[23] Scottish Hysteroscopy Audit Group. A Scottish audit of hysteroscopic surgery for menorrhagia: complications and follow up. BJOG: An International Journal of Obstetrics \& Gynaecology 1995;102(3):249-54.

[24] Propst AM, Liberman RF, Harlow BL, et al. Complications of hysteroscopic surgery: predicting patients at risk. Obstet Gynecol 2000;96(4):517-20.

[25] Jansen FW, Vredevoogd CB, van Ulzen K, et al. Complications of hysteroscopy: a prospective, multicenter study. Obstet Gynecol 2000;96(2):266-70.

[26] Deora S, Manchanda R, Ashraf S. Clinical audit of operative hysteroscopy and it's complications in a single institute performed by the same surgeon in the duration of four years. International Journal of Scientific Research 2018;7(1):25-6.

[27] Steptoe PC. Laparoscopy in gynaecology. $1^{\text {st }}$ edn. Edinburgh and London: E. \& S. Livingstone Ltd 1967: p. $40 \mathrm{~s}$.

[28] Hunt RB. Atlas of female infertility surgery. $2^{\text {nd }}$ edn. Year Book Medical Publishers 1986.

[29] Duffy S, Reid PC, Sharp F. In-vivo studies of uterine electrosurgery. Br J Obstet Gynaecol 1992;99(7):579-82. 